\title{
Contact and Collaboration Context Model
}

\author{
Siying Li, Marie-Hélène Abel \\ Sorbonne universités \\ Université de technologie de Compiègne, CNRS UMR \\ 7253, HEUDIASYC \\ 60203 Compiègne, France \\ \{siying.li, marie-helene.abel\}@utc.fr
}

\author{
Elsa Negre \\ Paris-Dauphine University, \\ PSL Research University, CNRS UMR 7243, LAMSADE \\ 75016 Paris, France \\ elsa.negre@dauphine.fr
}

\begin{abstract}
Working collaboratively is no longer an issue but a reality, what matters today is how to implement collaboration so that it is as successful as possible. It is therefore necessary to consider the criteria to be taken into account to promote its effectiveness. As part of our work, we are interested in taking into account the collaboration context for this purpose and the role that contact can take in this context. We wondered about its definition, representation and exploitation. The latter must be able to be done at different stages of collaboration: before, during and after. In this article, we present the collaboration context model that we have established and show why and how it can be used to establish successful collaboration.
\end{abstract}

Keywords-context, collaboration, contact.

\section{INTRODUCTION}

Nowadays, collaboration between people, organizations and even countries promotes the progress and development of human society. Successful collaborations create extra values and benefit collaborators. However, successful collaboration, which is not easy, is conditioned in different manners.

Facing to different situations, collaborators must make appropriate adjustments. Moreover, sometimes they have no idea how to improve collaboration itself. As part of our work, we propose to use context (contextual information related to collaboration) to help them and to evaluate their collaboration.

Unfortunately, the concept, 'context', is very dependent on the field of application. In order to construct a context model specific to collaboration, we analyzed the researches that have been done regarding of the collaboration, the context as well as the role of contact management in this area.

In this article, we present the definition of the concepts of collaboration and context, as well as our proposed definition of the collaboration context. We discuss the role of the concepts of social contact and business contact. We then justify the collaboration context model that we have established and illustrated how to use it before concluding.

\section{COllaboration}

\section{A. Definition}

The noun Collaboration comes from the verb collaborate which is defined as following in the Merriam-Webster
Dictionarya: "to work jointly with others or together especially in an intellectual endeavor". In addition, Patel, Pettitt and Wilson [18] indicate that "collaboration involves two or more people engaged in interaction with each other, within a single episode or series of episodes, working towards the common goals". According to Suto and Patitad [22], "collaboration is a communication process" and they emphasize the functionalities of knowledge transfer in the process of collaboration.

Note that members of collaboration can be individuals, groups of people or even organizations, for example, commercial collaborations between several companies or institutions. The members of collaboration are called collaborators.

The actions of collaborators to achieve the common goal are collaborative actions. The latter are ultimately carried out by human actors contributing to the collaboration as individual members, or members belonging to the group collaborators or organization collaborators.

It is specified that a collaborative action can generate one or more products as a result, and that from the analysis of all the actions carried out, certain regularities and techniques can be identified.

Finally, the definition of collaboration that we retain is the following:

Collaboration involves at least two collaborators and consists of a set of actions carried out by the human actors acting on behalf of the corresponding collaborator, in order to achieve a common goal.

\section{B. Discussion}

In order to qualify a successful collaboration, different factors must be considered. We will discuss these according to works of literature, in the following part.

To guide designers of collaboration systems, Briggs et al. [4] developed the Seven Layer Model of Collaboration (SLMC). Each layer is focus on one key area of concern. The Seven Areas of Concern for Designers of Collaboration Systems are: "Goal (desired state or outcome); Product (tangible or intangible artifact or outcome produced by the group's labor); Activity (sub-tasks that yield the products that

\footnotetext{
${ }^{\text {a }}$ https://www.merriam-webster.com/dictionary/collaborate
} 
constitute attainment of the group goal); Pattern of Collaboration (observable regularities of behavior and outcome that emerge over time in teamwork); Technique (reusable procedure for invoking useful interactions among people working towards a group goal, for example, brainstorming); Tool (artifacts or apparatus used in performing an operation for moving a group toward its goals); and Script (everything team members say to each other and do with their tools to move toward the group goal)."

The seven layers are linked with each other. Thus, the collaborators use tools to implement techniques enabling them to carry out activities (set of actions) to achieve a goal or a product. In doing so, scripts are established and patterns of collaboration are set up, which constitute the backbone of collaboration and allow it to be characterized (Briggs et al. [4]).

Wouters, Creff, Bella and Koudri [23] identify four prerequisites for successful collaboration: "1) A shared objective between involved stakeholders; 2) A synchronization of actions; 3) An exchange of information, between the right people, at the right time; 4) A complementarity between skills."

The SLMC (Briggs et al. [4]) and the prerequisites proposed by Wouters, Creff, Bella and Koudri [23] aim to make collaboration as successful as possible by identifying critical elements. These two works can be used to qualify a successful collaboration or not. We will see how they complement each other.

The first prerequisite integrates the first two layers of the SLMC. The actors of the collaboration members implicitly share the two areas, goal and product, of SLMC.

The second and third prerequisites are associated to the layers of SLMC: activity (set of actions), tool and technique. However, they do specify the layers. The actions must be synchronous. As for the exchange of information, it must take place between the relevant actors at the right time.

The final prerequisite is not even considered in the SLMC.

On the other hand, the SLMC accounts for scripts that can be considered as traces of activities. These traces are useful for establishing patterns of collaboration.

The necessary knowledge transfer for collaboration (Murphy, Stapleton and Smith [15]) can be achieved through information exchange in a broad sense. With the help of recorded scripts, it becomes possible to study these exchanges and analyse whether the carried out actions reflect the good application of the actors' knowledge. Then the arising question concerns the profile of the actors in collaboration: what knowledge, skills do they possess before the collaboration? How do they evolve during collaboration?

Establishing a profile of actors in collaboration is thus connected to the fourth pre-requisite of Wouters, Creff, Bella and Koudri [23]. Such a profile would make it possible to take other characteristics into consideration that could intervene to promote successful collaboration. Information exchanges will be more effective if the actors speak the same language, are familiar with the same collaboration techniques and/or tools, and appreciate each other (including networks).
In conclusion, qualifying a successful collaboration goes beyond reaching the shared goal. Various factors must be kept in mind. In some ways, these latter constitute the collaboration context. They focus on both the task to be completed and the resources in broad sense in order to carry it out: actors, tools, techniques, etc.

\section{CONTEXT}

\section{A. Definition}

"Context is a complex notion" (Adomavicius and Jannach [1]). According to Kofod-Petersen and Cassens [13], it is the key element used to help intelligent entities understand how occurrences in the surrounding world influence their own behaviors. What is more, "it stays a very ill-defined concept". (Bazire and Brézillon [3]) Its definitions in the literature are "too much dependent of their own contexts" (Bazire and Brézillon [3]) and "depending on the field of application" (Palmisano, Tuzhilin and Gorgoglione [17]), for example:

- "Context is the set of circumstances that frames an event or an object" (Bazire and Brézillon [3]). This definition is widely used in the field of psychology.

- "Context represents a set of explicit variables that model contextual factors in the underlying domain (for example, time, place, surroundings, device, occasion, and so on)." (Adomavicius and Tuzhilin [2]). This definition comes from recommender systems.

- \&Context is the set of environmental states and settings that either determines an application's behavior or in which an application event occurs and is interesting to the user." (Chen and Kotz [7]) This definition is generally used in the field of contextaware computing.

However, we retain a definition that seems to be a consensus on whatever the field of application: "context is any information that can be used to characterize the situation of an entity. An entity is a person, place, or object that is considered relevant to the interaction between a user and an application, including the user and applications themselves." (Dey [8]).

Information that could be considered in an entity's context is called contextual information. It represents the values of contextual factors (Adomavicius and Tuzhilin [2]). For example, for the 'time' factor, the value: ' $17 \mathrm{~h}$ ' is contextual information.

Multiple contextual factors are listed in the literature, such as "location, time, temperature or user identity" (Ryan, Pascoe and Morse [20]). Brown, Bovey and Chen [5] add another factor: people who are present with the user. In addition, there are "the state of people and groups" (Dey, Abowd and Salber [9]) in context-aware applications, "purchasing intent" (Palmisano, Tuzhilin and Gorgoglione [17]) in e-commerce, current events (Franklin and Flaschbart [11]) in intelligent environments, "space and location" for mobile systems (Rodden, Cheverst, Davies and Dix [19]). What's more, accessible devices and changes of these factors over time (Byun and Cheverst [6]) are also mentioned. 
Grouping contextual factors makes it possible to specify a measurable dimension. For example, a time dimension can include factors such as hours, minutes, seconds, time zone, and so on. Thus, an entity's context has the opportunity to become multidimensional.

The definition of an entity's context remains nevertheless dependent on the use of which we would like to make. It is therefore necessary to identify the appropriate dimensions as well as the associated factors. These latter are used to define the characteristics of an entity. Such features can be used not only to describe the entity at a given time $t$ but also to "infer possible actions and information needs" (Kofod-Petersen and Cassens [13]). For example, in forecasting weather, day j's context is used to predict the weather for the next few days.

In conclusion, after having synthesized these various works, we propose to define an entity's context by completing Dey[8]'s definition as follows:

Context is any information that can be used to characterize the situation of an entity over a given period of time. An entity is a person, place, event or object that is considered relevant to the interaction between a user and an application, including the user and the applications themselves.

\section{B. Discussion}

In this discussion we delve deeper into the definition of contextual information and in particular its dimensions and associated factors. We will then study the role that the notion of contact can hold in that regard.

Zimmermann, Lorenz and Oppermann [24] exploit five basic context categories (/dimensions) of an entity: "Individuality (Properties and attributes describing the entity itself); Time (Time coordinates of the entity); Location (Spatial coordinates of the entity); Activity (Tasks that the entity is currently and in future involved in); and Relation (Information about relations that the entity has established to other entities)." They construct a context model, which can be used for different types of entities: natural, human, artificial, and group entity.

As for Sladić and Milosavljević [21], they use the following dimensions: Actor, Action, Resource, Means, Time, Place and Objective. Negre [16], after having identified 10 dimensions of contextual factors: Time, Individuality, Activity, Relation, Place, Object, Season, Temperature, Social Context and Material Context, retains only 5 in the context of data warehouses (Time, Individuality/User Profile, Activity, Relations, Material Context). Moreover, Ferdousi, Negre and Colazzo [10] propose to analyze the context in 3 families: the physical context (containing 4 dimensions - temporal, spatial, environmental, and equipment), the personal context (and its 4 dimensions - demographic, social, psychophysiological, and cognitive) and the technical context (with 2 dimensions hardware and data).

By comparison, Zimmermann, Lorenz and Oppermann [24] do not take into account the influence of resources used by an entity during an activity. Negre [16] improves this by taking peripheral resources into account through the Material Context dimension. Sladić and Milosavljević [21] and Ferdousi, Negre and Colazzo [10] proceed further by proposing a Resource/Data dimension. From our point of view, this dimension of context is significant and would allow information to be inferred. Moreover, due to the rapid development of information and communication technologies, digital resources and metadata must be included. For example, a meeting report/note is a text resource that can contain several contextual information about the meeting, such as the purpose, time, location, participants,...

What about the notion of contact in the dimensions identified? Generally, this notion can have different meanings (Meanings are obtained in the Merrian-Webster ${ }^{\mathrm{b}}$ dictionary and Cambridge $^{c}$ dictionary) of which:

- State or action of people who are in contact, who communicate with each other, who are seeing each other: Maintaining contact with childhood friends.

- Information about a person who can help to carry out an action or obtain something (his profile: name, address, telephone, skills, etc.).

In the first meaning, it is necessary to note the presence of exchanges between people. Whereas in the second case, it is only information. What's more, it becomes possible to share a contact from its directory with his contact information.

Many systems have been developed to facilitate communication between people and the sharing of information about people. These include social media (Facebook, Twitter, Google+, LinkedIn), email management tools (Outlook, Gmail), real-time messaging software (MSN, Facebook Messenger, WeChat), etc. Using such systems provides the ability to define individual or group contacts. It is also possible to categorize these contacts, for example professional or personal contact. With the help of these systems, social or collaborative activities between users could be traced and can therefore be studied for different purposes (Liu [14]).

Taking the work of Negre [16] and Zimmermann, Lorenz and Oppermann [24], two dimensions are concerned by the notion, contact, according to the definitions we have retained:

- The Relation dimension considers contact in terms of connections or relationships between people. Ferdousi, Negre and Colazzo [10] address on the Social dimension. Therefore, the contact is directly tied to the activities carried out and the relationships they maintain with other people.

- The Individuality dimension considers contact from the point of view of the information characterizing the person who can help to carry out an action or obtain something.

Finally, it appears that the dimensioning of the context is not solid. The main reason is the complexity and dependence of the context. Another is that contextual information has a lifetime and its importance shifts over time. Moreover, they can be either dynamic or static, which depends on the time. For

\footnotetext{
${ }^{\mathrm{b}}$ https://www.merriam-webster.com/dictionary/contact

${ }^{\mathrm{c}}$ https://dictionary.cambridge.org/dictionary/english/contact
} 
example, during the same month, a person's age is relatively static. However, if he/she is moving to a new place during the month, then its address is dynamic. Therefore, from a contextual point of view, the address is more "important" than the age for the given month. In a project, the strength of contact between participants will be stronger during the duration of the project. It will be lower if there has been no interaction between people for a long time.

\section{Collaboration CONTEXT}

When we speak of context, it is necessarily associated with an entity: the context of what? As we have seen in Section III, the context specifies the contextual dimensions of an entity that it describes. In the following, we explore the contextual dimensions to be considered when the entity being described is collaboration. We will talk about the collaboration context.

\section{A. Definition}

If we speak of a collaborative context, literally the entity in the definition in Section III.A is collaboration. Its context contains characteristic information that is highly dynamic and evolves over time.

\section{Precisely the definition becomes:}

The collaboration context is any information that can be used to characterize the situation of collaboration over a given period of time. Here, collaboration is an event considered relevant to the interaction between a user and an application, including the user and the applications themselves.

Based on the definition of collaboration given and discussed in Section II, contextual information of collaboration includes its members, common goal, activities (set of collaborative actions) done, period of time, tools, techniques, scripts, patterns used and products. All the information are gathered into factors or dimensions to construct a collaboration context model.

Resuming the discussion in Section III.B, a contact in the

Fig. 1. Collaboration Context Model

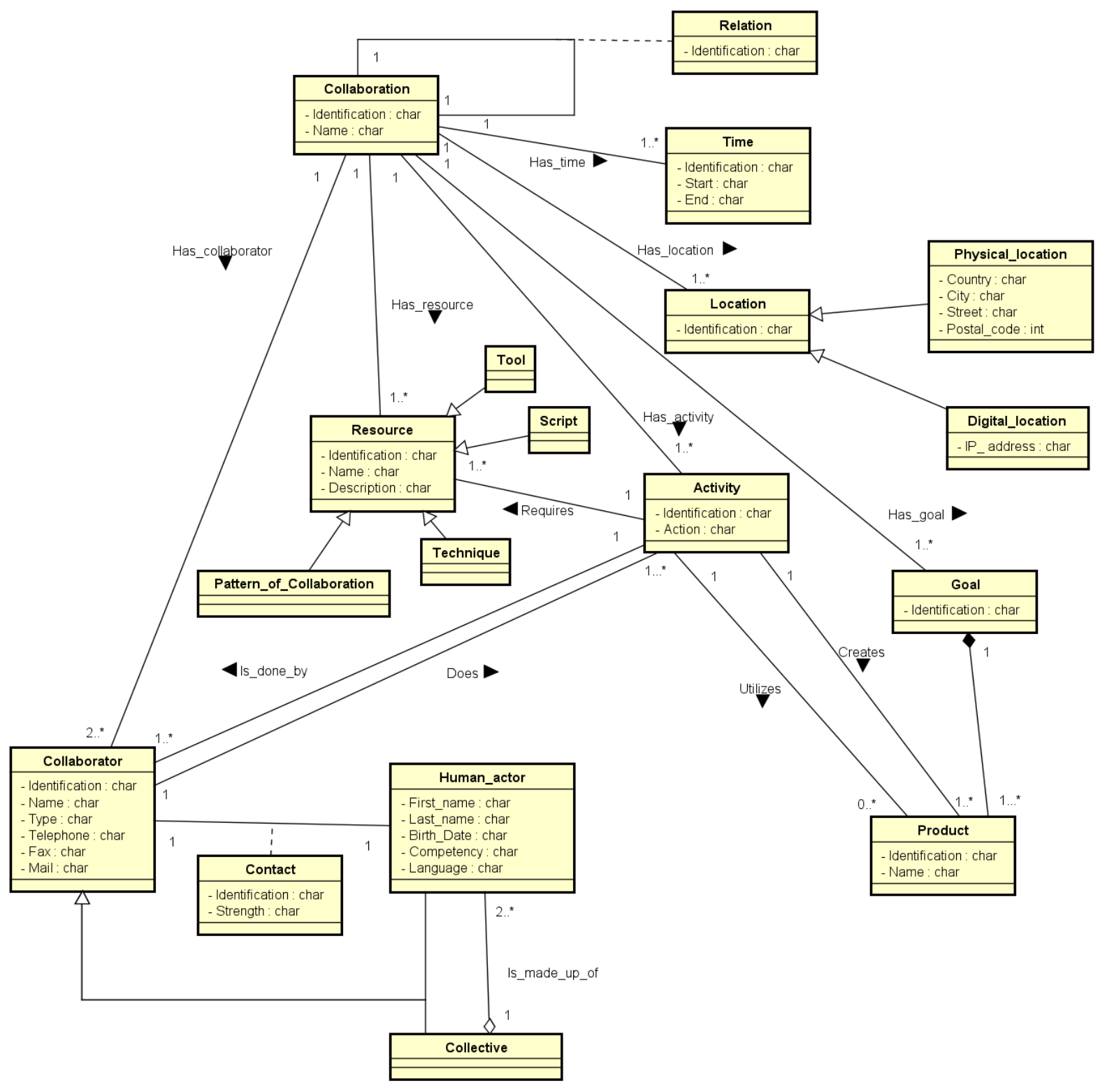


collaboration context will be represented as:

- A person acting as an intermediary, messenger, connection or special information source.

- Relations between collaborators tied to the activities they have carried out together.

\section{B. Modeling}

We use the above definition of the collaboration context to establish its model and focus on three principal interrelated concepts: contextual information, factor and dimension.

As discussed in Section 3.1, contextual information is the value of a contextual factor. These factors can be clustered into contextual dimensions to describe an entity's situation. These three contextual concepts can constitute a general architecture of the context model. The fundamental dimensions defined by Zimmermann, Lorenz and Oppermann [24] respect this architecture and can be used for different types of entities.

The construction of collaboration context model, therefore, can be based on the architecture presented above. It is then a matter to define the dimensions, their factors and their value domains in accordance with why we want to use them: to be able to establish and measure the successfulness of collaboration.

With regard to collaboration, using the definition and discussion in section II, the characteristic information to reflect the situation of a collaboration is summarized as follows: collaborator, activity (set of actions), human actors, goals, patterns of collaboration, techniques, tools, scripts and products

Collaborators, as members of a collaboration, can differ according to whether they are individual (person) or collective (group or organization). Collaborators, as members of a collaboration, can differ according to whether they are individual (person) or collective (group or organization). The collaborators share the same goal. Human actors, carry out activities (set of actions) and use tools, techniques on behalf of the collaborator. Then scripts and collaboration patterns can be observed. The activities implemented produce results (outputs) to achieve the final outcome: the shared goal.

In the context, using the definition and discussion in Section III, eight dimensions are retained: Time, Location, Activity, Relation, Contact, Collaborator, Resource and Goal. The first fours are directly inspired by the work of Zimmermann, Lorenz and Oppermann [24]. The others allow to identify the members of a collaboration as well as to specify the resources they will exploit within the collaboration and the common goal. (See Fig. 1).

In this model we have clearly defined the concept, collaboration, which takes the individuality dimension of Zimmermann, Lorenz and Oppermann [24] in some ways. The goal is defined as a dimension to enable it to be related to the products that will be created or used by the activities carried out by the collaborators. The latter are therefore modelled as such and a dimension is thus dedicated to them. Resources are used to undertake these activities, hence this dimension is created, namely, Resource. Contacts can be solicited by querying the relation Contact: the case of a contact defined as a person (Human_actor). Contacts also can occur between collaborators at the time of the realization of an activity, which will be modelled using the relation Is_done_by. Finally, having created the concept collaboration allows us to consider the relationships among the collaborations in the form of a dimension, Relation.

Note that factors in dimensions can be translated as simple attributes/values or as properties. The collaboration pattern factor consists of recording traces of interaction between collaborators. Having modelled the concept, collaborator, also makes it possible to integrate the profile of the collaborator, whether in the form of a human actor or a group/collective (groups of human actors).

\section{Usage and Exploitation Scenario}

In this section, we discuss the value of the collaboration context model established in the perspective of successful collaboration. This model can thus be used as a prelude, a guide, or a review of a collaboration. We illustrate our purpose on the basis of a scenario.

\section{1) Prelude of a collaboration}

In order to establish a collaboration, it is necessary to identify or define its characteristics. The collaboration context model established, through the intelligence of the dimensions and factors retained, can serve as a support for it. A collaboration is being prepared: what is the goal of the collaboration? What is the expected duration of it? Who will be the collaborators? What tasks/activities will be assigned to them? Successful collaboration depends on these identifications. As long as the goal is well determined, it becomes easier to identify the skills needed and the collaborators to be recruited with such skills. Choosing the collaborators is a matter of selecting those who seem best able to work well together, those who use the same working language, the same working tools and techniques, those who have already collaborated, those who have already made contact with each other and established relations, etc. The use of contact information can be very beneficial in identifying collaborators.

\section{2) Guide of a collaboration}

As the process of collaboration proceeds, some indicators can be used to identify blocking points. Are the planned activities being carried out on time? Do collaborators interact regularly? If there is a problem, do they communicate with each other to resolve it? These indicators can be obtained by observation/study of interaction traces recorded from the model. And it becomes possible to set up a strategy to deal with the issues that cause the poor value in such indicators. The use of established contacts can facilitate the interpretation of indicators as well as the establishment of a solution.

\section{3) Review of a collaboration}

When the collaboration is over, it is always interesting to learn lessons from its progress and results obtained. It is fairly easy to measure whether the goal has been achieved, but measuring the successfulness of collaboration is more difficult. This kind of measurement can be done by studying the review of the collaboration, which includes all the activities carried out. 
These activities allow connecting to resources, products, contacts and collaborators in a task of collaboration. It is also possible to measure the quantity and frequency of actions carried out between which collaborators and with which tools. In addition, this model allows updating the contacts of a collaborator. Using these measurements and crossing them with the profile of the collaborators, it becomes possible to advance analysis of the collaboration and draw conclusions in order to plan new collaborations.

As a result, the use of our collaboration context model permits to use tangible elements to evaluate a collaboration according to several dimensions, such as resources, collaborators, their contacts and activities. This evaluation aims to characterize a collaboration with a degree of successfulness.

\section{CONCLUSION}

In this article, we focus on the concept, collaboration context, which enables to qualify collaboration. Based on the literature of collaboration and context, we have established a definition of collaboration context. We investigated the role that contacts could play in it. We then constructed a model based on an architecture of an entity's context model. We finally explained why and how our collaboration context model, taking into account contacts, could be used to set up a collaboration (its prelude), its implementation (support for its development, guide) and the analysis of its review.

Our research perspectives include the development of this model in a digital environment, as well as that of a recommender system to exploit it. The recommendations could then be static during the prelude phase of the collaboration and the review. They could also be done dynamically as the collaboration progresses. Ultimately, further work would be needed on the relations that such a model maintains with that of the user's context (or the person/actor's context).

\section{REFERENCES}

[1] G. Adomavicius and D. Jannach, "Preface to the special issue on context-aware recommender systems," User Modeling and UserAdapted Interaction, vol. 24, pp. 1-5, February 2014.

[2] G. Adomavicius and A. Tuzhilin, "Context-Aware Recommender Systems," Recommender Systems Handbook, Springer, Boston, MA, pp. 191-217, 2011.

[3] M. Bazire and P. Brézillon, "Understanding context before using it," In International and Interdisciplinary Conference on Modeling and Using Context, Springer, Berlin, Heidelberg, pp. 29-40, 2005.

[4] R.O. Briggs, G. Kolfschoten, G.J.D. Vreede, C. Albrecht, D.R. Dean and S. Lukosch, "A seven-layer model of collaboration: Separation of concerns for designers of collaboration systems," ICIS 2009 Proceedings, p.26, 2009.

[5] P.J. Brown, J.D. Bovey and X. Chen, "Context-aware applications: from the laboratory to the marketplace," IEEE personal communications, volume 4, pp. 58-64, October 1997.

[6] H.E. Byun and K. Cheverst, "Utilizing context history to provide dynamic adaptations," Applied Artificial Intelligence, Taylor \& Francis, Byun, H.E, volume 18, p. 533-548, July 2004.
[7] G. Chen and D. Kotz, "A survey of context-aware mobile computing research," Technical Report, Dartmouth College, Hanover, NH, USA, 2000.

[8] A.K. Dey, "Understanding and using context," Personal and ubiquitous computing, Springer-Verlag, volume 5, number 1, pp. 4-7, Januaray 2001.

[9] A.K. Dey, G.D. Abowd and D. Salber, "A conceptual framework and a toolkit for supporting the rapid prototyping of context-aware applications," Human-computer interaction, L. Erlbaum Associates Inc , volume 16, number 2, pp. 97-166, December 2001.

[10] Z. Ferdousi, E. Negre and D. Colazzo, "Context Factors in ContextAware Recommender System," in AISR 2017 Atelier interdisciplinaire sur les systèmes de recommandation, Paris, France, May 2017.

[11] D. Franklin and J. Flaschbart, "All gadget and no representation makes jack a dull environment," In Proceedings of the AAAI 1998 Spring Symposium on Intelligent Environments, pp.155-160, March 1998.

[12] B. Guo, D. Zhang, D. Yang, Z. Yu, and X. Zhou, "Enhancing memory recall via an intelligent social contact management system," IEEE Transactions on Human-Machine Systems, volume 44, number 1, pp. 78-91, Febraury 2014.

[13] A. Kofod-Petersen and J. Cassens, "Using activity theory to model context awareness," In Modeling and Retrieval of Context, Springer, Berlin, Heidelberg, pp. 1-17, 2006.

[14] C.L. Liu, "Contact information management system architecture for social media," In Computer Communications and Networks (ICCCN), 2011 Proceedings of 20th International Conference, p. 1-5, July 2011.

[15] F. Murphy, L. Stapleton and D. Smith, "Tacit Knowledge and human centred systems: The key to managing the social impact of technology," International Multitrack Conference of Advances in Control systems, University of Vienna (TUWien), Austria, 2004.

[16] E. Negre, "Prise en compte du contexte dans les systèmes de recommandations de requêtes OLAP," EDA 2017, BI \& Big Data, p. 110, May 2017.

[17] C. Palmisano, A. Tuzhilin and M. Gorgoglione, "Using context to improve predictive modeling of customers in personalization applications," IEEE transactions on knowledge and data engineering, volume 20, number 11, pp. 1535-1549, November 2008.

[18] H. Patel, M. Pettitt and J.R. Wilson, "Factors of collaborative working: A framework for a collaboration model," Applied ergonomics, volume 43, number 1, pp. 1-26, Janurary 2012.

[19] T. Rodden, K. Cheverst, K. Davies and A. Dix, "Exploiting context in hci design for mobile systems," In Workshop on human computer interaction with mobile devices, Glasgow, pp. 21-22, May 1998.

[20] N. Ryan, J. Pascoe and D. Morse, "Enhanced reality fieldwork: the context aware archaeological assistant," Bar International Series, volume 750, p. 269-274, 1999.

[21] G. Sladić and B. Milosavljević, "Context-Aware Access Control for IoT Driven Processes," In the 8th PSU-UNS International Conference on Engineering and Technology (ICET-2017), Akdeniz University, Antalya, Turkey, 2017.

[22] H. Suto and P. Patchanee, "A representation model of collaboration in design process," In Control Conference (ASCC), 2015 10th Asian, pp. $1-5,2015$.

[23] L. Wouters, S. Creff, E.E. Bella and A. Koudri, "Collaborative systems engineering: Issues \& challenges," In Computer Supported Cooperative Work in Design (CSCWD), 2017 IEEE 21st International Conference on, p. 486-491, 2017.

[24] A. Zimmermann, A. Lorenz and R. Oppermann, "An operational definition of context," In International and Interdisciplinary Conference on Modeling and Using Context, Springer, Berlin, Heidelberg, pp. 558$571,2007$. 